\title{
Optimizing levodopa therapy for Parkinson's disease with levodopa/carbidopa/entacapone: implications from a clinical and patient perspective
}

\section{David J Brooks}

Division of Neuroscience, Faculty of Medicine, Imperial College, Hammersmith Hospital, London, UK
Correspondence: David J Brooks Hartnett Professor of Neurology, Imperial College, Cyclotron Building, Hammersmith Hospital, Du Cane Road, WI 2 ONN, London, UK

Tel +44208383 3I 72

Fax +44 $208383 \quad 1783$

Email david.brooks@csc.mrc.ac.uk

\begin{abstract}
After 40 years of clinical experience, levodopa remains the gold standard treatment for Parkinson's disease (PD) despite the recent emergence of a host of new therapies. Some physicians are cautious when prescribing levodopa because of its association with motor complications. Evidence now suggests that levodopa-associated complications are a result of deep troughs in delivery of levodopa to the brain caused by the short plasma half-life of conventional levodopa formulations (levodopa and a dopa decarboxylase inhibitor [DDCI]). Dosing strategies, such as dose increases and dose fractionation, may be effective in the short term. For the longer-term, levodopa/carbidopa/entacapone provides pharmacokinetically optimized levodopa therapy that significantly increases the plasma half-life and bioavailability of levodopa, providing more consistent plasma levodopa levels without deep troughs. Evidence from clinical trials in PD patients experiencing re-emergence of symptoms due to wearing-off has consistently shown that levodopa/DDCI and entacapone significantly increases ON-time and affords greater functionality, as measured by the Unified Parkinson's Disease Rating Scale (UPDRS) with conventional levodopa. These trials have also shown that levodopa/DDCI and entacapone is generally well tolerated, with notable adverse events including worsening dyskinesia, nausea and diarrhea. Patients experiencing re-emergence of symptoms due to wearing-off may benefit from optimized levodopa therapy with levodopa/carbidopa/entacapone.
\end{abstract}

Keywords: levodopa, wearing-off, dyskinesia, entacapone, Stalevo

\section{Introduction}

Parkinson's disease (PD) is a chronic and progressive neurodegenerative condition associated with considerable morbidity and social and economic consequences. The prevalence of PD increases with age and, since life expectancy is rising worldwide, the global burden of care for the condition is likely to increase markedly over the next 25 years. A recent study estimated that in 2005 there were over 1 million individuals with PD in Western Europe and the USA; this value was projected to more than double by 2030 with dramatic rises expected in developing eastern countries (Dorsey et al 2007). Therefore, there is an ever greater need to continue the development of effective management strategies for PD.

Historically, PD has been viewed as a motor disease with cardinal symptoms of resting tremor, rigidity and bradykinesia (Litvan et al 2003). In recent years there has been an increasing awareness of the importance of non-motor symptoms, such as mood and anxiety disorders, fatigue and apathy, cognitive impairments, sleep disorders and addictions (Chaudhuri et al 2006). These non-motor symptoms can be as debilitating as motor symptoms and contribute to severe disability, impaired quality of life and shortened life expectancy. Optimal PD management should, therefore, encompass 
both dopaminergic replacement therapy, treatment of non-motor problems, such as coexistent dementia, depression and autonomic dysfunction, and physical intervention through exercise, nutritional advice, speech therapy and psychosocial support for both patient and carer. Together, these elements help to maintain the patient's functional capacity and quality of life.

\section{Levodopa - gold standard therapy for Parkinson's disease}

The choice of pharmacotherapy for PD has increased dramatically over the last 25 years as the understanding of the pathogenesis of PD has improved. Until the 1960s, drug treatment for PD was limited to anticholinergics, which had low efficacy and a high incidence of associated gastrointestinal and neuropsychiatric side effects (Katzenschlager et al 2003). The introduction of levodopa had a dramatic impact, significantly reducing disability and mortality and increasing patient quality of life (Maier Hoehn 1983). Despite the emergence of a host of new dopaminergic therapies for PD, including dopamine agonists, dopamine reuptake inhibitors, and MAO-B inhibitors, levodopa remains the gold standard treatment for PD 40 years on.

A number of well-controlled, large-scale, long-term clinical trials have consistently demonstrated the superior symptomatic control of conventional levodopa formulations (levodopa and a dopa decarboxylase inhibitor [DDCI]) compared with dopamine agonists. The comparison of the agonist pramipexole with levodopa on motor complications of Parkinson's disease (CALM-PD) trial compared treatment with conventional levodopa (levodopa/carbidopa, 100/25 mg) 3 times/day with pramipexole $(0.5 \mathrm{mg}) 3$ times/day (Holloway et al 2004). At 4-year follow-up, treatment with levodopa was associated with a significant improvement in the total Unified Parkinson's Disease Rating Scale (UPDRS) score from baseline versus pramipexole (mean change 2 vs -3.2 points, respectively; treatment effect 5.9 points; $\mathrm{p}=0.003$ ). Similar results were reported in the 5-year 056 study, which compared the safety and efficacy of conventional levodopa (50 mg once daily to $400 \mathrm{mg} 3$ times/day) with ropinirole $(0.25-8 \mathrm{mg}$ 3 times/day) (Rascol et al 2000). In this study, treatment with levodopa was associated with a significantly greater improvement in the UPDRS III (motor) score compared with ropinirole (mean change 4.8 vs 0.8 points, respectively; treatment effect 4.48 points; $\mathrm{p}=0.008$ ). There was also a trend towards improvement in the UPDRS II (activities of daily living $[\mathrm{ADL}]$ ) with levodopa, although the difference between treatment groups was not significant $(\mathrm{p}=0.08)$.
Poorer functionality observed with dopamine agonists versus levodopa in the CALM-PD and 056 studies occurred despite the option of supplemental levodopa therapy in both trials. The recent pergolide-versus-L-dopa-monotherapyand-positron-emission-tomography (PELMOPET) study analyzed the efficacy and safety of a third dopamine agonist, pergolide (0.75-5.0 mg/day), against conventional levodopa (150-1200 mg/day) using a strict monotherapy design that did not allow for levodopa rescue (Oertel et al 2006). At 3 -year follow-up, the mean change in total UPDRS score from baseline was improved by 6.4 points in the levodopa treatment arm, but had deteriorated by 2.1 points in the pergolide arm $(p<0.001)$. It should be noted that pergolide is no longer available in the United States due to its association with an increased risk of cardiac fibrosis. Nevertheless, the substantial between-treatment difference in the PELMOPET study serves to highlight the superiority of levodopa over dopamine agonists in terms of symptomatic control. This may reflect the fact that dopamine agonists act primarily on D2 receptor subtypes, whereas exogenous dopamine generated from levodopa will act at both D1 and D2 dopamine sites.

\section{The limitations of levodopa}

Despite the demonstrated efficacy of treatment with levodopa, some physicians are cautious when prescribing the drug because of its association with the emergence of motor complications (Marsden and Parkes 1977). The first randomized clinical trial of conventional levodopa (ELLDOPA), which was carried out relatively recently, showed that high doses of levodopa/DDCI are a factor in the development of motor complications (Fahn 2005). Complications can emerge as early as 5 to 6 months after treatment initiation with doses $\geq 600 \mathrm{mg} /$ day (Fahn 2005). Wearing-off and dyskinesia associated with long-term conventional levodopa therapy can result in disability and have a significant impact on a patient's quality of life (Chapuis et al 2005). In a limited number of cases, usually young, severely-affected patients, motor complications can outweigh the functional benefits provided by treatment. Consequently, initiation of levodopa may be postponed in an attempt to delay the onset of these complications.

The rationale for initiating $\mathrm{PD}$ therapy with dopamine agonist monotherapy in early disease is based on a reduction in the risk of dyskinesias. Results from the levodopacontrolled trials of dopamine agonists consistently show a marked reduction in the incidence of dyskinesia with agonist therapy. In the CALM-PD study, pramipexole treatment resulted in a significant reduction in the risk of developing 
dyskinesia (24.5\% vs 54\%; p < 0.001) and wearing-off $(47 \%$ vs $62.7 \% ; \mathrm{p}=0.02$ ) compared with levodopa (Holloway et al 2004). However, the incidence of clinically relevant "disabling dyskinesias" at 4 years follow-up was very low (4\% vs $7 \%$ ) and did not differ significantly between the treatment groups. In the 056 study, ropinirole was associated with a significant reduction in the risk of developing dyskinesia compared with levodopa ( $\mathrm{p}<0.001)$ with an incidence of $20 \%$ vs $45 \%$ at 5 years; the rate of wearing-off was also lower with ropinirole (23\% vs 34\%) (Rascol et al 2000).

However, the lower efficacy of the dopamine agonists means that the vast majority of patients eventually require the superior symptomatic control of supplemental levodopa. In the CALM-PD study, $72 \%$ of patients randomized to pramipexole required levodopa supplementation at 4 years (Holloway et al 2004). In the 056 trial, $66 \%$ of patients in the ropinirole arm required levodopa with a mean daily dose of $427 \mathrm{mg}$ of adjunct levodopa by 5 years (Rascol et al 2000). It could be argued that keeping levodopa doses at such a level or lower may protect against onset of motor complications. However, a recent post-hoc analysis of the 056 trial revealed that once levodopa is added to ropinirole therapy, the risk of developing dyskinesias is not significantly different from that in patients initiated on levodopa therapy (Rascol et al 2006). Therefore, while ropinirole monotherapy delays the onset of dyskinesia compared with conventional levodopa, it does not exert any protective effect against levodopainduced dyskinesia when used as adjunctive therapy. The implication of this finding is that ropinirole does not address the cause of motor complications associated with long-term levodopa therapy.

\section{Deep troughs in levodopa delivery with conventional levodopa}

Mounting evidence now suggests that levodopa-associated motor complications are a result of high dosage and the noncontinuous delivery of levodopa to the brain. Conventional levodopa/DDCI is rapidly metabolized in the periphery, resulting in a short plasma half-life of $\sim 60-90$ minutes (Nutt and Fellman 1984). In the early stages of PD, this short plasma half-life is discordant with the clinical effect of the drug, which may persist for many hours, indicating that surviving dopaminergic neurons are able to store the exogenous dopamine generated from levodopa and so buffer variations in levodopa availability. However, with progression and further neuronal degeneration, the levodopa-buffering capacity is lost and deep troughs in plasma levodopa levels correspond with deep troughs in dopamine within the striatum, resulting in pulsatile stimulation of striatal dopaminergic receptors (de la Fuente-Fernandez et al 2001). Under these circumstances, patients experience a predictable pattern of symptom re-emergence that corresponds with these low troughs. Sustained pulsatile stimulation of dopaminergic receptors is also thought to underlie the pathogenesis of dyskinesia (Olanow et al 2006). Continuous enteral infusion of levodopa has been shown to significantly increase the bioavailability of levodopa and prevent low troughs in plasma levodopa levels (Stocchi 2005). The improved plasma profile achieved with infusion is associated with dramatic improvements in OFF-time and dyskinesia (Stocchi 2005). Such data support the theory that motor complications result from deficiencies in the delivery of levodopa due to the poor pharmacokinetic profile of conventional oral formulations.

\section{Dosing strategies with conventional levodopa formulations}

Because of the impracticality and considerable expense of enteral levodopa infusion, physicians generally rely on strategies designed to improve the delivery of oral levodopa to prevent motor complications. The standard approach is to alter the dosing regimen of conventional levodopa formulations, either by increasing the size of each levodopa dose or by "fractionating" the total daily levodopa dose into smaller, more frequent doses. While these modification strategies may be effective in the short term, they fail to address the issue of the short half-life of conventional levodopa in the longterm, and thus repeated modifications to the dosing strategy are often required.

Increasing the amount of levodopa administered at each dose does not eliminate troughs in plasma levels and may lead to an increased incidence and severity of dyskinesia (Figure 1) (Stocchi 2006). Levodopa dose fractionating is similarly ineffective at reducing troughs in plasma levels and is often associated with intermittent re-emergence of symptoms due to suboptimal levodopa exposure (Stocchi 2006).

\section{The influence of treatment adherence on levodopa efficacy}

Dose fractionation may also exacerbate the issue of deep troughs in levodopa delivery with conventional formulations through reduced adherence to treatment schedules. An observational study that analyzed treatment adherence by dispensing PD therapy into electronic monitoring bottles found that compliance with treatment regimens was low for PD (Grosset et al 2005). Adherence to the total dose was satisfactory ( $\geq 80 \%$ ) in $80 \%$ of patients, while the remaining 
(A)

$100 \mathrm{mg}$; 3-hour intervals

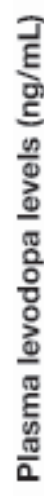

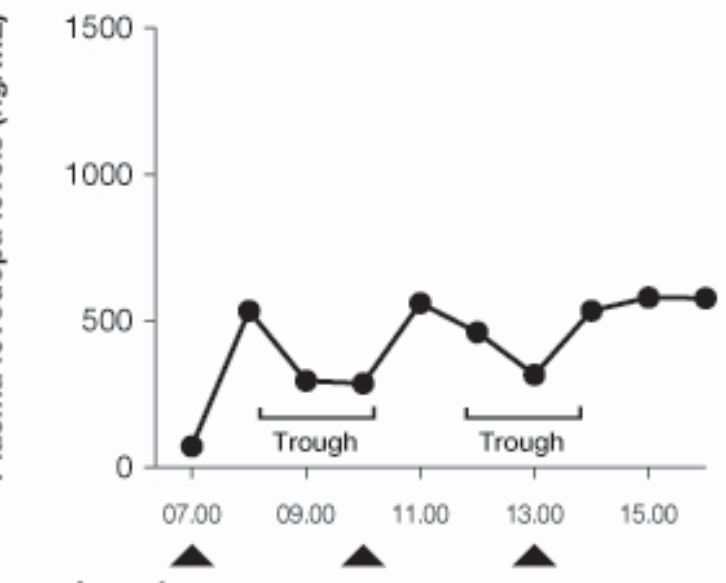

Levodopa administration
$100 \mathrm{mg}$ levodopa
$150 \mathrm{mg}$; 3-hour intervals

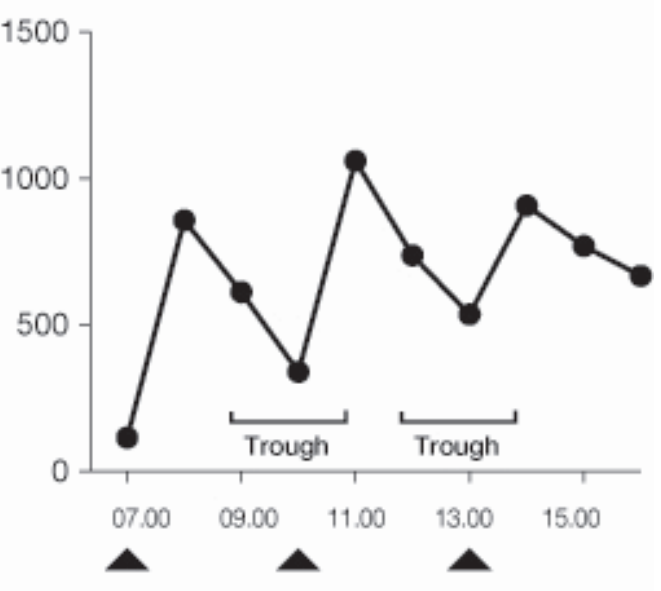

Time of day $\quad 150 \mathrm{mg}$ levodopa

(B)

\section{5-hour intervals}

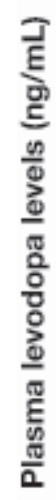

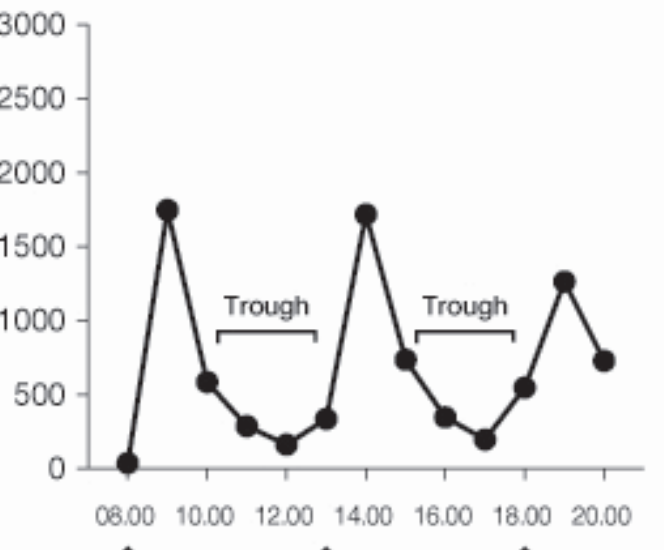

Levodopa

administration

\section{3-hour intervals}

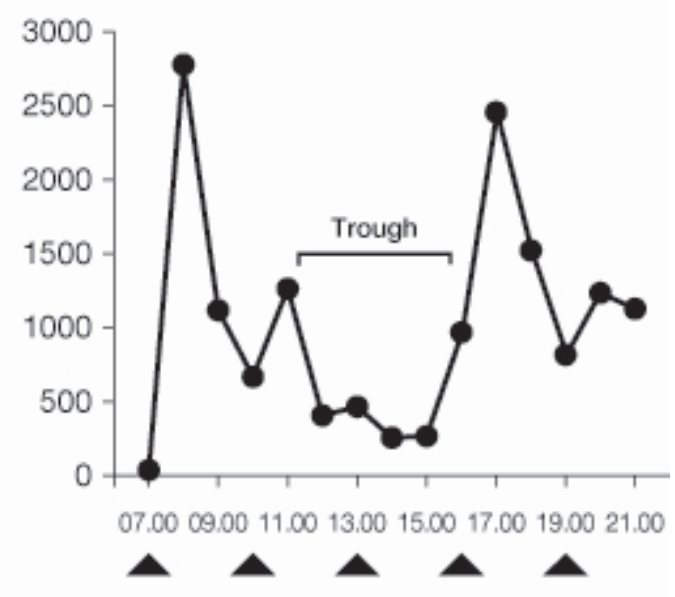

Time of day

Figure I Plasma levodopa profiles with conventional levodopa dosing strategies. Schematic depicting plasma levodopa levels in Parkinson's disease patients experiencing re-emergence of symptoms due to wearing-off achieved by (A) increasing the daily levodopa/carbidopa dose from $300 \mathrm{mg} / \mathrm{day}$ to $450 \mathrm{mg} / \mathrm{day}$; (B) increasing the frequency of levodopa/carbidopa dosing from 3 times/day to 5 times/day.

$20 \%$ of patients were found to be under-users. However, timing of medication was poor regardless of whether patients' adherence to treatment was satisfactory or unsatisfactory, suggesting that erratic drug taking is the norm rather than the exception in PD.

Poor adherence to treatment regimens was significantly associated with younger age, lower quality of life, higher depression scores and, notably, with taking more antiparkinsonian tablets per day (Grosset et al 2005). Therefore, increasing the number of daily doses by fractionation is likely to reduce treatment adherence. While non-adherence to long-acting drugs may have a lesser impact, the potency of levodopa combined with its short half-life is likely to accentuate the effect of a missed dose and create extended periods of sub-therapeutic levodopa levels. The proposed association between non-continuous delivery of levodopa and treatment-associated motor complications suggests that dose fractionation may actually contribute to, rather than prevent, these problems in a subset of patients. Rather than adjusting the administration of conventional 
levodopa formulations, the goal of oral levodopa therapy should be to optimize the pharmacokinetics of the drug and extend its duration of effect, and so provide similar benefits to those seen with enteral infusion of levodopa.

\section{Optimizing the delivery of levodopa Erratic absorption with controlled- release levodopa}

In recognition of the limitations of dose adjustment strategies, oral, controlled-release (CR) levodopa preparations were developed in the late 1980 s to provide smoother, more continuous plasma levels of levodopa with fewer daily doses. These formulations use a degradable polymer matrix to retard the release of levodopa from the tablet into the gut. However, clinical experience with CR levodopa preparations has revealed erratic absorption and unpredictable plasma levels (Nyholm et al 2003). A significant delay to ON-time is a common feature of CR formulations, and dose failure may also occur. A clinical study that evaluated the effects of CR levodopa/carbidopa (Sinemet $\mathrm{CR}^{\circledR}$, Bristol-Myers Squibb Company, NJ, USA) compared with the conventional immediate-release formulation in levodopa-naïve patients found no relative therapeutic benefit with twice-daily CR levodopa/carbidopa and no significant difference in the rates of motor complications between the treatment groups (Koller et al 1999). Similarly, data from a clinical study of identical design evaluating CR levodopa/benserazide (Madopar $\mathrm{CR}^{\circledR}$, Roche Products Ltd, Welwyn Garden City, UK) over 5 years also indicated no relative therapeutic benefit with CR levodopa (Dupont et al 1996). Currently, use of CR levodopa formulations is limited and usually confined to night-time administration.

\section{Levodopa/carbidopa/entacapone: optimized levodopa therapy}

Levodopa/carbidopa/entacapone (Stalevo ${ }^{\circledR}$, Novartis Pharmaceuticals Corporation, NJ, USA) is a pharmacokinetically optimized levodopa formulation that peripherally inhibits both of the main pathways of levodopa metabolism (Silver 2004). Compared with conventional levodopa, the pharmacokinetic profile of levodopa with dual enzyme inhibition is markedly improved, increasing the half-life of levodopa by up to $85 \%$ and the bioavailability of the drug by $35 \%$ in plasma (Stalevo 2007). In patients with PD, transferring from a conventional levodopa to levodopa/carbidopa/entacapone is associated with significantly higher trough levels, reflecting a more consistent levodopa plasma profile (Figure 2) (Muller et al 2006). Positron emission tomography (PET) studies with a positron-emitting analog of levodopa ( ${ }^{18} \mathrm{~F}-6$-L-dopa) reveal that levodopa/ carbidopa and entacapone significantly increases striatal levodopa delivery and storage by up to $50 \%$ in patients with $\mathrm{PD}$, compared with conventional levodopa/carbidopa (Sawle et al 1994). Therefore, the pharmacokinetic data would suggest that levodopa/carbidopa/entacapone may provide more consistent delivery of levodopa to the brain, so providing increased symptomatic benefits with fewer motor complications.

\section{Clinical experience with levodopa/ dopa decarboxylase inhibitor and entacapone}

Four randomized, double-blind, 6-month, Phase III clinical trials have evaluated the efficacy and safety of levodopa/DDCI and entacapone therapy compared with levodopa/DDCI and placebo in PD patients with motor fluctuations (Parkinson Study Group 1997; Rinne et al 1998; Poewe et al 2002; Brooks and Sagar 2003; Larsen et al 2003). Data from these trials demonstrate that the improved pharmacokinetic profile of levodopa/DDCI and entacapone therapy translates into greater efficacy, providing significant benefits in terms of symptom control compared with conventional levodopa. In the study by Rinne et al, levodopa/DDCI and entacapone therapy significantly increased the proportion of ON-time by $16 \%$ (treatment effect +1.4 hours vs placebo; $p<0.001$ ) and, correspondingly, reduced OFF-time by $24 \%$ (treatment effect -1.3 hours vs placebo; $\mathrm{p}<0.001$ ) over the 6-month treatment period (Stalevo 2007). This increased duration of self-reported symptomatic control was reflected in functional benefits of ADL and motor control as measured by UPDRS sub-scales II and III, respectively (Figure 3). There were no significant differences between treatment arms in the UPDRS I sub-scale (mentation, behaviour and mood). However, improvements in both UPDRS II and III sub-scales, and the total UPDRS score were significantly greater with levodopa/DDCI and entacapone than with levodopa/DDCI and placebo $(\mathrm{p}<0.05)$ (Figure 3 ). These benefits were achieved despite a $12 \%$ reduction in the mean daily levodopa dose, from $701 \mathrm{mg}$ at baseline to $614 \mathrm{mg}$ at 6 months. Over the same time period the mean daily levodopa dose increased by $2 \%$ in the control group, resulting in a difference of $102 \mathrm{mg} /$ day between the groups at the end of the study ( $\mathrm{p}<0.001)$ (Rinne et al 1998).

Levodopa/DDCI and entacapone therapy was generally well tolerated; the adverse events reported significantly $(\mathrm{p}<0.05)$ more frequently than in the control group were nausea (20\% vs $8 \%$, respectively), diarrhea (20\% vs $7 \%$, 


\section{Levodopa/carbidopa/entacapone -๑-Levodopa/carbidopa}

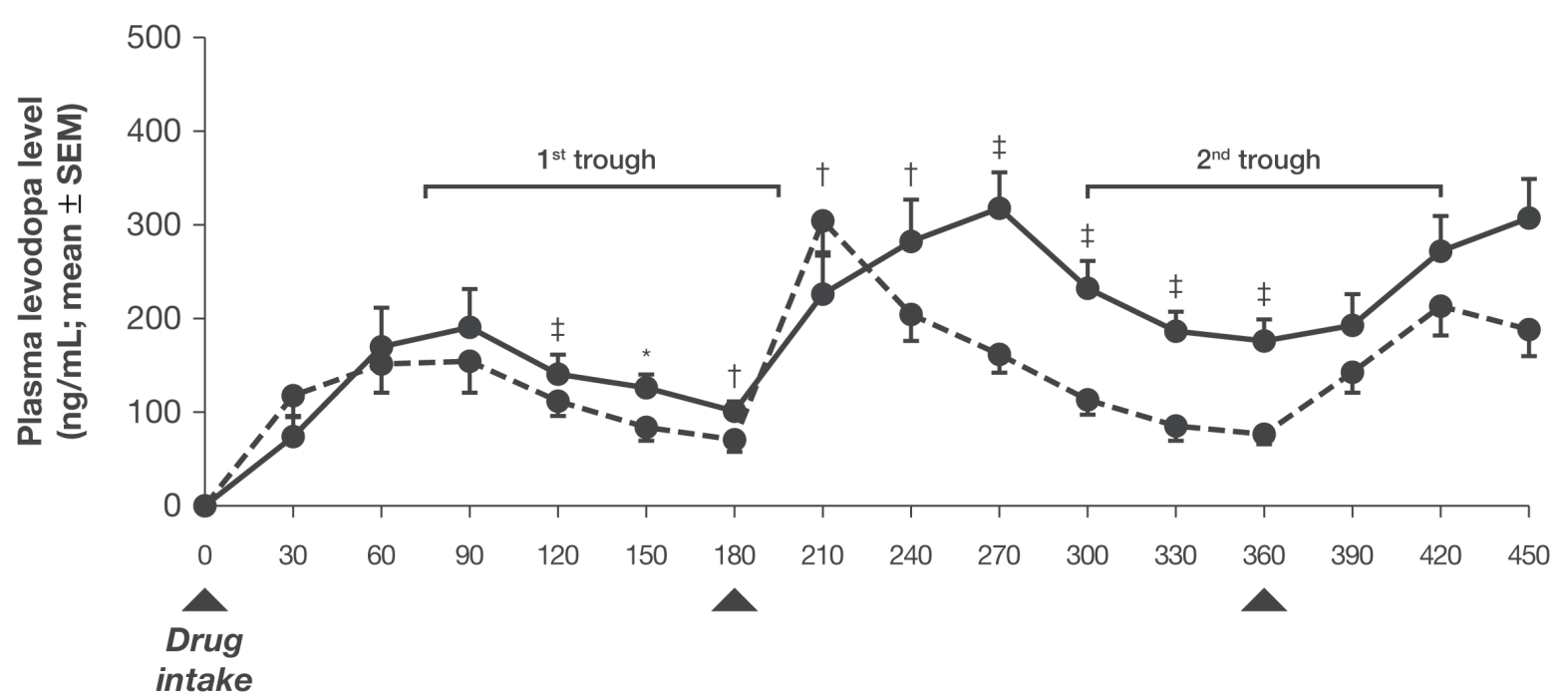

Time (minutes)

Figure 2 Plasma levodopa profile with levodopa/carbidopa/entacapone versus levodopa/carbidopa. Treatment with levodopa/carbidopa/entacapone 3 times/day significantly improves troughs in plasma levodopa levels compared with equivalent doses of levodopa/carbidopa in patients with Parkinson's disease; ${ }^{*} \mathrm{p}<0.05$; ${ }^{\dagger} \mathrm{P}<0.0 \mathrm{I} ;{ }^{\ddagger} \mathrm{p}<0.00 \mathrm{I}$; SEM, standard error of the mean.

respectively), harmless discoloration of urine (11\% vs $1 \%$, respectively) and worsening of dyskinesia ( $8 \%$ vs $1 \%$, respectively) (Rinne et al 1998). The incidence of disabling or painful dyskinesias was not increased and the dyskinesias that did occur could be effectively managed with dose reductions. No patients withdrew from the study due to dyskinesia. Similar results were achieved in the major trials of levodopa/DDCI and entacapone with adverse events summarized in Table 1 (Parkinson Study Group 1997; Rinne et al 1998; Myllyla et al 2001; Poewe et al 2002; Brooks and Sagar 2003).
Long-term follow-up of the study in a single-arm, open-label extension revealed that the symptomatic benefits and tolerability of levodopa/DDCI and entacapone therapy were maintained at 3 years (Larsen et al 2003). As in the double-blind phase, UPDRS II and III scores improved in the initial stages of treatment and remained comparable with baseline levels at 3-year follow-up (Figure 4). These measurements, conducted during patients' ON-time, indicate that levodopa/DDCI and entacapone maintained the quality of ON-time in PD patients with motor fluctuations. Furthermore, the mean daily levodopa dose remained below baseline levels for the duration of the

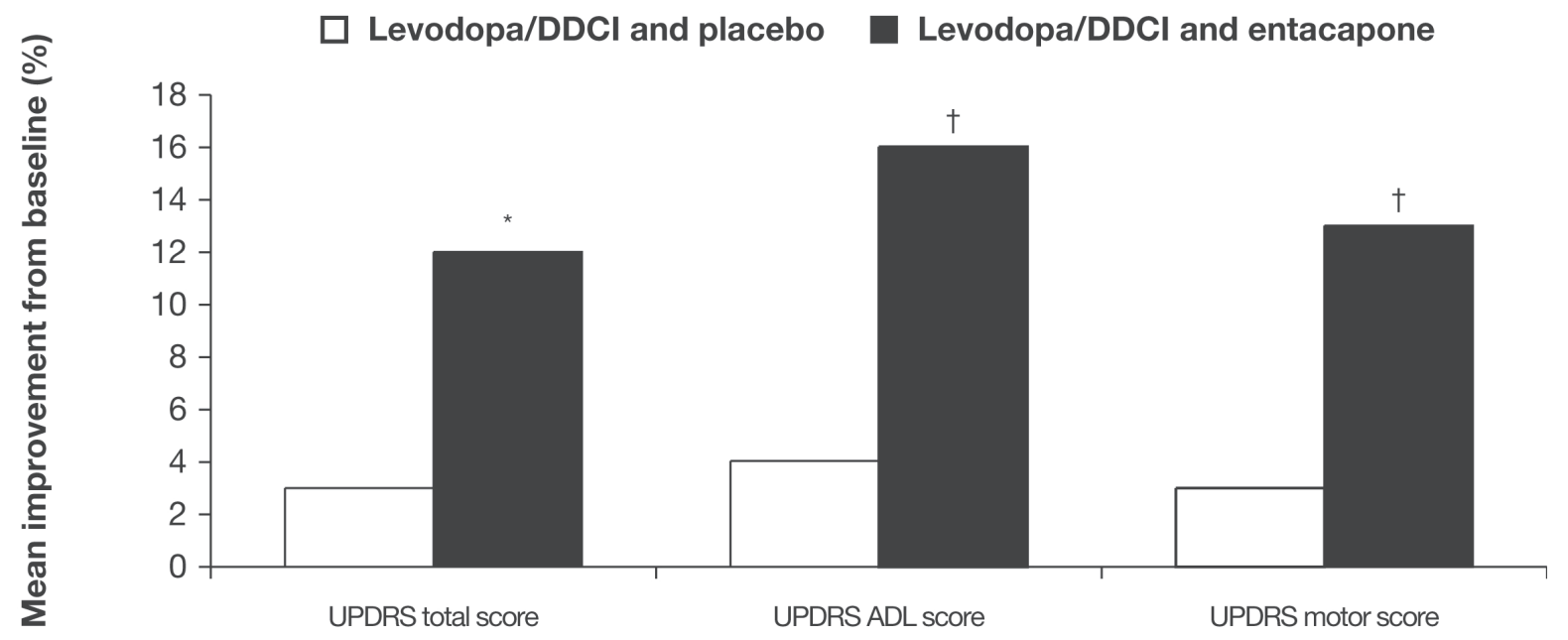

Figure 3 Efficacy of levodopa/dopa decarboxylase inhibitor and entacapone therapy in the short-term. Treatment with levodopa/DDCl and entacapone is associated with significant improvements in functional control as determined by UPDRS total, ADL and motor scores compared with levodopa/DDCl and placebo over 6 months. ${ }^{*} \mathrm{p}<0.0 \mathrm{I} ;{ }^{\dagger} \mathrm{p}<0.05$ Abbreviations: UPDRS, Unified Parkinson's Disease Rating Scale; ADL, activities of daily living; DDCl, dopa decarboxylase inhibitor. 
Table I Most frequent adverse events associated with levodopa/ dopa decarboxylase inhibitor and entacapone therapy

\begin{tabular}{lll}
\hline Adverse event & Patients (\%) \\
\cline { 2 - 3 } & $\begin{array}{l}\text { Levodopa/DDCI } \\
\text { and entacapone } \\
(\mathbf{n}=\mathbf{8 0 6})\end{array}$ & $\begin{array}{l}\text { Levodopa/DDCI } \\
\text { and placebo } \\
(\mathbf{n}=\mathbf{4 9 7})\end{array}$ \\
\hline Dyskinesia & 30.4 & 17.5 \\
Nausea & 13.6 & 7.4 \\
Urine discoloration & 10.8 & 0 \\
Diarrhea & 10.3 & 3.8 \\
Abdominal pain & 9.6 & 5.6 \\
Vomiting & 7.3 & 4.2 \\
Constipation & 7.2 & 4.2 \\
Fatigue & 6.1 & 3.6 \\
\hline
\end{tabular}

Table based on analysis of data in the SEESAW $(n=205)$, NOMECOMT $(n=171)$, FILOMEN $(\mathrm{n}=326)$, CELOMEN $(\mathrm{n}=30 \mathrm{I})$ and UK-IRISH $(\mathrm{n}=300)$ studies (Parkinson Study Group 1997; Rinne et al 1998; Haapaniemi et al 200I; Myllyla et al 2001; Poewe et al 2002; Brooks and Sagar 2003).

Abbreviations: $\mathrm{DDCl}$, dopa decarboxylase inhibitor.

3-year follow-up, and remained significantly lower at 3 years versus baseline (696 vs $737 \mathrm{mg}$ /day, respectively; $\mathrm{p}=0.0034$ ) (Larsen et al 2003). The most frequent adverse events reported in the study were insomnia (30\%), dizziness (20\%), nausea $(20 \%)$ and aggravation of parkinsonian symptoms (17\%). A total of $14 \%$ of patients discontinued treatment due to an adverse event, with diarrhea, hallucinations and abdominal pain the most frequent reasons.

\section{When to optimize levodopa therapy}

It has recently been suggested that normalization of basal ganglia function through earlier initiation of dopaminergic therapy may delay clinical progression and provide long-term functional benefits (Schapira and Obeso 2006). This theory is supported by data from a retrospective pooled analysis of three comparably designed, double-blind, Phase III studies (Parkinson Study Group 1997; Rinne et al 1998; Poewe et al 2002) and their long-term, open-label extensions (Larsen et al 2003) conducted in PD patients with motor fluctuations on conventional levodopa. The analysis divided patients into two groups: those who received levodopa/DDCI and entacapone during the 6-month double-blind phase (early start), and those who received levodopa/DDCI and placebo in the double-blind phase but moved over to levodopa/DDCI and entacapone in the open-label extension (delayed start). At 1-year follow-up, patients in the early start group reported a significantly greater improvement in the UPDRS III score (1.33 points) compared with patients in the delayed start group (Nissinen et al 2006). This study is the first to indicate that earlier initiation of optimized levodopa therapy may have additional benefits for patients on conventional levodopa and experiencing re-emergence of symptoms due to wearing-off, and that delaying such therapy might adversely affect long-term patient benefit.

\section{Efficacy of levodopa/carbidopal entacapone in patients with stable Parkinson's disease}

Levodopa/carbidopa/entacapone is indicated for the treatment of patients with PD experiencing a re-emergence of their symptoms due to wearing-off; however, its effects have also been evaluated in patients with stable PD (ie non-fluctuating disease) (Poewe et al 2002; Brooks and Sagar 2003). In the subset of stable patients included in the Celomen study group

\section{Baseline $\mathbf{3}$ years}

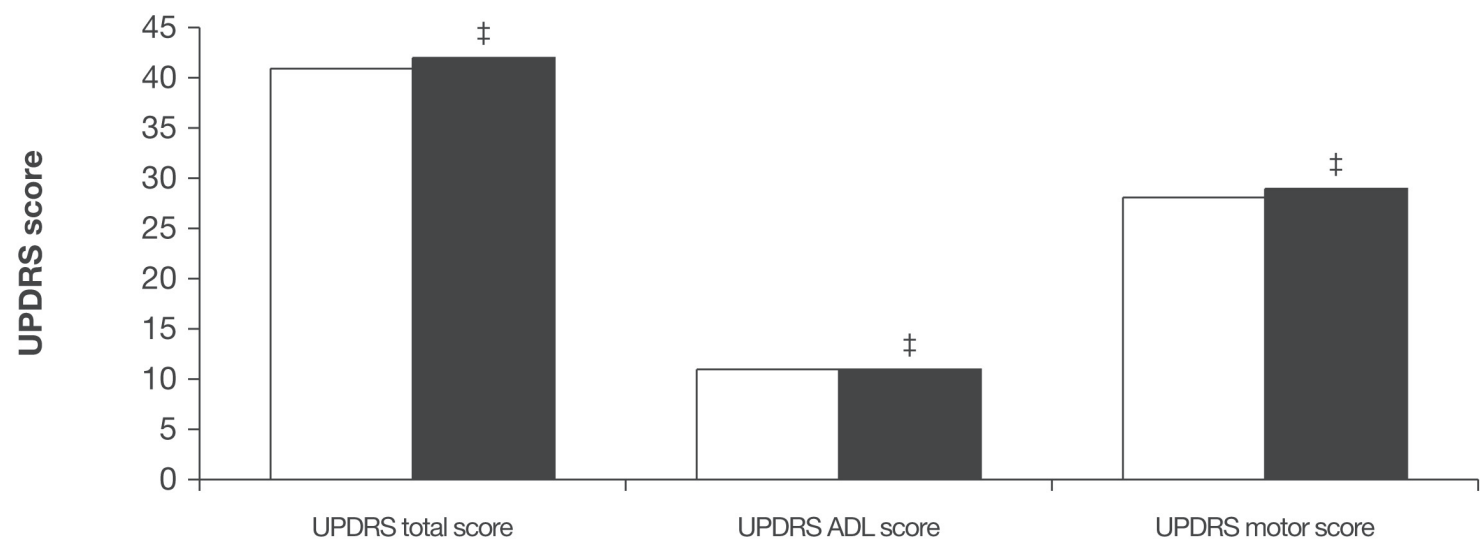

Figure 4 Efficacy of levodopa/dopa decarboxylase inhibitor and entacapone therapy in the long-term. Functionality is maintained over three years with levodopa/DDCl and entacapone.

${ }^{\ddagger} \mathrm{p}=$ not significant.

Abbreviations: UPDRS, Unified Parkinson's Disease Rating Scale; ADL, activities of daily living; DDCl, dopa decarboxylase inhibitor. 
(CELOMEN) trial, mean UPDRS II and III scores improved from baseline by 1.0 point and 2.3 points, respectively, at 6 months with levodopa/DDCI and entacapone (Poewe et al 2002). This is compared with a deterioration in both UPDRS measures with levodopa/DDCI and placebo, although the small sample size precluded meaningful statistical analysis of between-treatment differences. A similar trial designed to evaluate both unstable and stable patients observed no significant improvement in mean UPDRS III motor scores with levodopa/DDCI and entacapone in stable patients at 6 months. In contrast, UPDRS II ADL scores were significantly improved in stable patients treated with levodopa/DDCI and entacapone compared with those receiving levodopa/ DDCI and placebo ( $p<0.01)$ (Brooks and Sagar 2003).

\section{Increased quality of life with levodopa/ carbidopa/entacapone}

It has been suggested that quality of life assessments, which assess all aspects of a patient's experience of treatment, are a more relevant measure of a PD therapy than the range of rating tools currently employed (Wheatley et al 2002). Separate analysis of UPDRS scores and adverse events fails to empirically consider the competing benefits and risks of treatment. Quality of life measures may be particularly suitable when evaluating treatment in early PD when the extent of motor complications is low, which makes identification of changes in functionality difficult.
The recent QUEST-AP study, a randomized, double-blind, parallel-group design trial compared the efficacy of levodopa/ carbidopa/entacapone with levodopa/carbidopa in patients with stable PD (Fung 2006). The primary efficacy variable of this study was quality of life as measured by the Parkinson's disease questionnaire (PDQ)-8 scale. At 12 weeks, total quality of life was improved with levodopa/carbidopa/entacapone but deteriorated with levodopa/carbidopa, with a significant difference between the treatment groups $(-1.4$ points; $p=0.021)$. The treatment effect of levodopa/carbidopa/entacapone was particularly significant in the emotional wellbeing, social relationships, communication and stigma aspects of the PDQ-8 scale (Figure 5). Among secondary efficacy variables, significant reductions in the total and UPDRS II (ADL) scores were reported $(\mathrm{p}=0.047$ and $\mathrm{p}=0.032$, respectively).

\section{Conclusions}

After 40 years of clinical experience, levodopa remains the most effective method of managing the symptoms of PD. However, conventional levodopa formulations are often used sparingly by physicians concerned about the possibility of motor complications with long-term use. Regardless of initial treatment, the vast majority of patients with PD will eventually require the superior symptomatic control afforded by levodopa therapy. It is now widely recognized that the poor pharmacokinetic profile of conventional levodopa is an important factor in the development of motor complications. Consequently, there

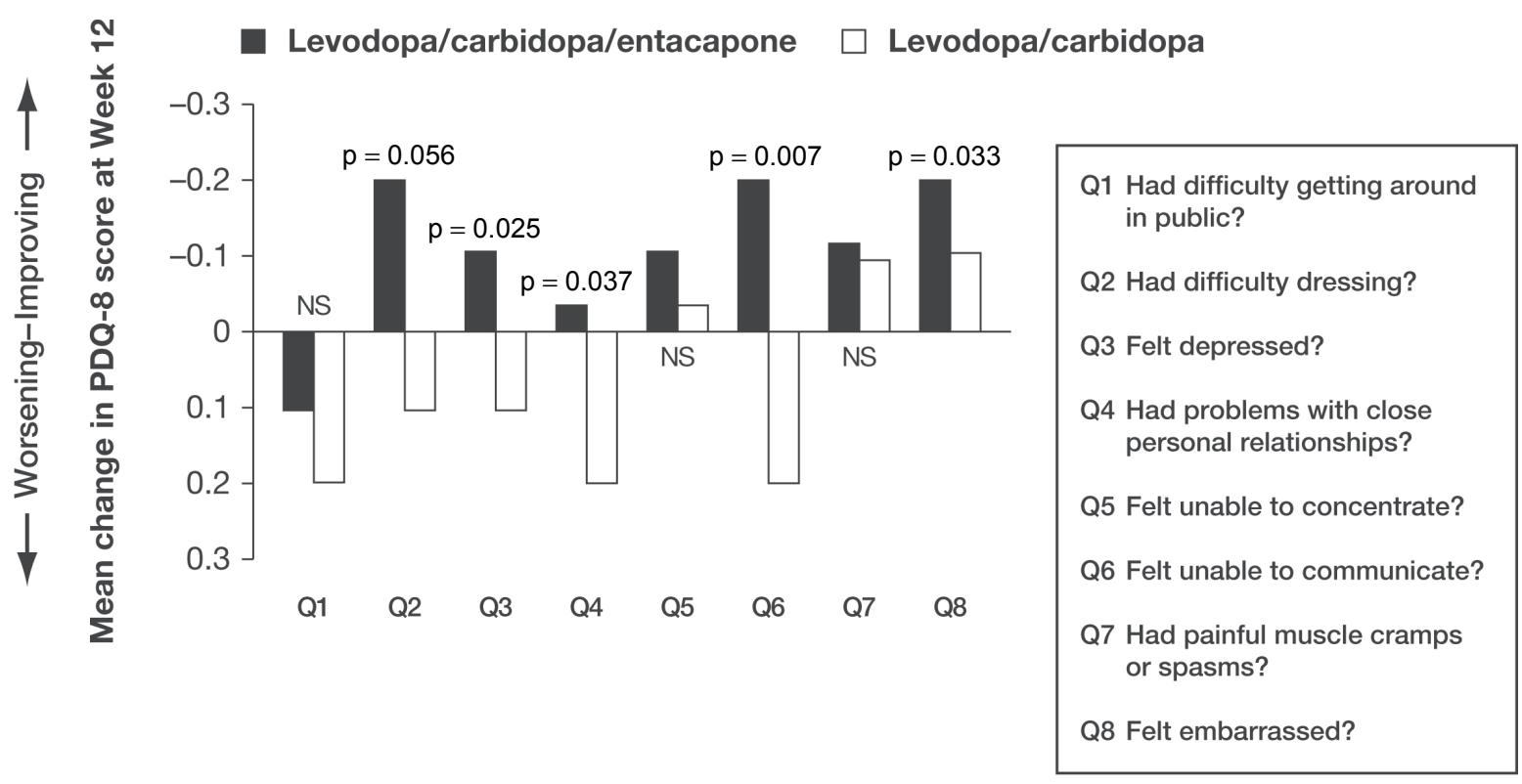

Figure 5 Effect of levodopa/carbidopa/entacapone on quality of life in patients with stable Parkinson's disease. Treatment with levodopa/carbidopa/entacapone is associated with significant benefits in terms of quality of life compared with levodopa/carbidopa, as determined by the Parkinson's disease questionnaire-8.

Abbreviations: PDQ, Parkinson's disease questionnaire; NS, not significant; Q, question. 
is a need for optimized oral levodopa therapy that overcomes the limitations of conventional levodopa formulations while providing effective symptomatic control.

Levodopa/carbidopa/entacapone is a pharmacokinetically optimized levodopa formulation that offers more consistent plasma levodopa levels without deep troughs. Clinical evidence in PD patients experiencing motor fluctuations with conventional levodopa demonstrates that treatment with levodopa/DDCI and entacapone significantly increases time with less symptoms and affords greater functionality, as measured by UPDRS and quality of life scales. These benefits are maintained for at least 3 years with no need to increase the mean daily levodopa dose over this period. Furthermore, earlier initiation of levodopa/DDCI and entacapone therapy may also provide functional benefits compared with delaying treatment. Levodopa/DDCI and entacapone is generally well tolerated, with the most notable adverse events observed in clinical trials being an increased incidence of dyskinesia, nausea and diarrhea. Overall, these results suggest that patients experiencing re-emergence of symptoms due to wearing-off may benefit from optimized levodopa therapy with levodopa/carbidopa/entacapone.

\section{References}

Brooks DJ, Sagar H. 2003. Entacapone is beneficial in both fluctuating and non-fluctuating patients with Parkinson's disease: a randomised, placebo controlled, double blind, six month study. J Neurol Neurosurg Psychiatry, 74:1071-9.

Chapuis S, et al. 2005. Impact of the motor complications of Parkinson's disease on the quality of life. Mov Disord, 20:224-30.

Chaudhuri KR, et al. 2006. Non-motor symptoms of Parkinson's disease: diagnosis and management. Lancet Neurol, 5:235-45.

de la Fuente-Fernandez R, et al. 2001. Biochemical variations in the synaptic level of dopamine precede motor fluctuations in Parkinson's disease: PET evidence of increased dopamine turnover. Ann Neurol, 49:298-303.

Dorsey ER, et al. 2007. Projected number of people with Parkinson disease in the most populous nations, 2005 through 2030. Neurology, 68:384-6.

Dupont E, et al. 1996. Sustained-release Madopar HBS compared with standard Madopar in the long-term treatment of de novo parkinsonian patients. Acta Neurol Scand, 93:14-20.

Fahn S. 2005. Does levodopa slow or hasten the rate of progression of Parkinson's disease?. J Neurol, 252:iv37-42.

Fung V. 2006. A randomized, double - blind study to compare the effect on quality of life of levodopa/carbidopa/entacapone (Stalevo) with levodopa/carbidopa in patients with Parkinson's disease with no or minimal, non-disabling motor fluctuations. Movement Disorder Society of Australia Clinical Research and Trials Group (MDSCRTG) and QUEST-AP Study Group. Mov Disord, 21:S446 Abstract \#37.

Grosset KA, et al. 2005. Suboptimal medication adherence in Parkinson's disease. Mov Disord, 20:1502-7.

Haapaniemi H, et al. 2001. Tolerability and safety of entacapone in the treatment of Parkinson's disease. Parkinsonism Relat Disord, 7:S57.

Holloway RG, et al. 2004. Pramipexole vs levodopa as initial treatment for Parkinson disease: a 4-year randomized controlled trial. Arch Neurol, 61:1044-53.

Katzenschlager R, et al. 2003. Anticholinergics for symptomatic management of Parkinson's disease. Cochrane Database Syst Rev, CD003735.
Koller WC, et al. 1999. Immediate-release and controlled-release carbidopa/ levodopa in PD: a 5-year randomized multicenter study. Carbidopa/ Levodopa Study Group. Neurology, 53:1012-9.

Larsen JP, et al. 2003. The tolerability and efficacy of entacapone over 3 years in patients with Parkinson's disease. Eur J Neurol, 10:137-46.

Litvan I, et al. 2003. Movement Disorders Society Scientific Issues Committee report: SIC Task Force appraisal of clinical diagnostic criteria for Parkinsonian disorders. Mov Disord, 18:467-86.

Maier Hoehn MM. 1983. Parkinsonism treated with levodopa: progression and mortality. J Neural Transm Suppl, 19:253-64.

Marsden CD, Parkes JD, 1977. Success and problems of long-term levodopa therapy in Parkinson's disease. Lancet, 1:345-9.

Muller T, et al. 2006. Inhibition of catechol-O-methyltransferase contributes to more stable levodopa plasma levels. Mov Disord, 21:332-6.

Myllyla V, et al. 2001. Twelve-month safety of entacapone in patients with Parkinson's disease. Eur J Neurol, 8:53-60.

Nissinen H, Kuoppamaki M, Leinonen M. 2006. Early initiation of entacapone leads to superior 5-year efficacy compared to delayed initiation in Parkinson's disease patients receiving traditional levodopa/DDCI therapy. Mov Disord, 21:S593.

Nutt JG, Fellman JH. 1984. Pharmacokinetics of levodopa. Clinical Neuropharmacology, 7:35-49.

Nyholm D, et al. 2003. Optimizing levodopa pharmacokinetics: intestinal infusion versus oral sustained-release tablets. Clin Neuropharmacol, 26:156-63.

Oertel WH, et al. 2006. Pergolide versus levodopa monotherapy in early Parkinson's disease patients: The PELMOPET study. Mov Disord, 21:343-53.

Olanow CW, et al. 2006. Continuous dopamine-receptor treatment of Parkinson's disease: scientific rationale and clinical implications. Lancet Neurol, 5:677-87.

Parkinson Study Group. 1997. Entacapone improves motor fluctuations in levodopa-treated Parkinson's disease patients. Parkinson Study Group. Ann Neurol, 42:747-55.

Poewe WH, et al. 2002. Efficacy and safety of entacapone in Parkinson's disease patients with suboptimal levodopa response: a 6-month randomized placebo-controlled double-blind study in Germany and Austria (Celomen study). Acta Neurol Scand, 105:245-55.

Rascol O, et al. 2000. A five-year study of the incidence of dyskinesia in patients with early Parkinson's disease who were treated with ropinirole or levodopa. 056 Study Group. N Engl J Med, 342:1484-91.

Rascol O, et al. 2006. Development of dyskinesias in a 5-year trial of ropinirole and L-dopa. Mov Disord, 21:1844-50.

Rinne UK, et al. 1998. Entacapone enhances the response to levodopa in parkinsonian patients with motor fluctuations. Nomecomt Study Group. Neurology, 51:1309-14.

Sawle GV, et al. 1994. The effect of entacapone (OR-611) on brain [18F]-6-L-fluorodopa metabolism: implications for levodopa therapy of Parkinson's disease. Neurology, 44:1292-7.

Schapira AH, Obeso J. 2006. Timing of treatment initiation in Parkinson's disease: a need for reappraisal?. Ann Neurol, 59:559-62.

Silver DE. 2004. Clinical experience with the novel levodopa formulation entacapone + levodopa + carbidopa (Stalevo). Expert Rev Neurother, 4:589-99.

Stalevo. 2007. US prescribing information [online]. Accessed March 2007. URL: http://www.pharma.us.novartis.com/product/pi/pdf/ stalevo.pdf

Stocchi F. 2005. Optimising levodopa therapy for the management of Parkinson's disease. J Neurol, 252:IV43-8.

Stocchi F. 2006. The levodopa wearing-off phenomenon in Parkinson's disease: pharmacokinetic considerations. Expert Opin Pharmacother, 7:1399-407.

Wheatley K, et al. 2002. Evaluating drug treatments for Parkinson's disease: how good are the trials?. BMJ, 324:1508-11. 
